\title{
ESTRATEGIA PEDAGÓGICA PARA EL ANÁLISIS DE LOS ODS EN UN PROGRAMA DE NEGOCIOS INTERNACIONALES ${ }^{1}$
}

\author{
María Isabel Guerrero Molina \\ maria.guerrerool@amigo.edu.co \\ Universidad Católica Luis Amigó
}

Resumen

Los objetivos de Desarrollo Sostenible analizan problemáticas basadas en las buenas prácticas que cada nación debe implementar para mitigar los impactos de los indicadores que se presentan a nivel mundial; los delegados y representantes diplomáticos de las naciones señalan estrategias de mejoramiento en la Asamblea General realizada cada año. En la investigación, se estableció como objetivo general, analizar la aplicación de la técnica didáctica de juego rol como estrategia pedagógica para la enseñanza de los Objetivos de Desarrollo Sostenible en un programa académico, relacionado con los negocios internacionales, se aplicó una metodología cualitativa de tipo descriptivo, a través de dos eventos y en cada uno de ellos se evaluaron tres fases y 16 variables, se aplicó una rúbrica de evaluación con una valoración numérica y con preguntas abiertas, aplicado a 152 participantes matriculados en la asignatura de Diplomacia y Relaciones Internacionales en el periodo 2018. Para la recolección de la información secundaria se utilizaron bases de datos especializadas y fuentes oficiales de las Naciones Unidas.

Los hallazgos se enfocan en el mejoramiento de las estrategias aplicadas y se encuentra como resultado, que la aplicación de diferentes metodologías de aprendizaje para la aprehensión de conocimientos, se constituyen como una alternativa eficaz y eficiente; de esta manera se mejoran y se fortalecen las habilidades y fortalezas para cada participante, robusteciendo el trabajo colaborativo y el trabajo individual como soporte del aprendizaje.

\section{Palabras clave}

Desarrollo de las habilidades, juego de simulación, método de enseñanza, negociación.

\footnotetext{
1 Producto derivado del proyecto: Dinámicas Exportadoras de las Pymes Rad. 53004-09 de febrero de 2018 y estrategias pedagógicas: Simulación de la Asamblea General de la Organización de las Naciones Unidas - ONU. Universidad Católica Luis Amigó.
} 


\section{Introducción}

Los Objetivos de Desarrollo Sostenible - ODS, son un compendio de buenas prácticas adoptado por los miembros constitutivos de la Organización de las Naciones Unidas - ONU; en el año 2015, los líderes mundiales se comprometieron a cumplir en un periodo de 15 años, las 17 temáticas que aquejan las naciones a nivel global. Estos indicadores, se proyectan con la expectativa de ser cumplidos, para ello, se establecen acciones de mejoramiento en el cronograma gubernamental y así, seguir de cerca los avances de cada uno de ellos; desde la academia, se desea aportar a la resolución de estos desde la educación y el aprendizaje de las causas y efectos que cada uno representa en la sociedad.

La problemática que se aborda desde el programa de Negocios Internacionales estudiado en la ciudad de Medellín se centra, en el papel que deben cumplir las instituciones de educación superior al momento de enseñar y de transmitir los conocimientos a los estudiantes del programa que se encuentran cursando como proceso de formación.

Los negocios internacionales son dinámicos y cambiantes, esto amerita que el docente/tutor y el estudiante/participante estén a la vanguardia de los sucesos a nivel global, con el objetivo de poder realizar una lectura de la realidad según los hechos y el material informativo disponible a través de las bases de datos y de la información que brindan las fuentes oficiales para proponer una 
estrategia pedagógica que dé respuesta a las necesidades de formación de los estudiantes participantes.

El programa de Negocios Internacionales se orienta en brindarle a los estudiantes experiencias de formación que permitan que cada participante analice, comprenda y reflexione acerca de la realidad de las instituciones políticas y privadas de Colombia, y de los países que presentan indicadores alarmantes frente a los Objetivos de Desarrollo Sostenible. Fueron analizados en tres eventos pedagógicos, a través de la asignatura de Diplomacia y Relaciones Internacionales; en los periodos académicos entre 2018 y 2019, los objetivos analizados fueron Acción por el clima (13), Fin de la pobreza (1) y Hambre cero (2).

La implementación de una estrategia pedagógica, consiste en educar a los participantes sobre temas de actualidad y política internacional, por medio de la técnica didáctica juego de simulación o de intervención de roles basado en la representación de delegados representantes de los diferentes países que intervienen, por medio de debates y ejercicios basados en negociaciones internacionales, entorno a las problemáticas mundiales, y la representación de los diferentes órganos gubernamentales, delegados y representantes de las Naciones Unidas.

Por medio de este juego didáctico, los participantes mejoran las competencias relacionadas con la resolución y manejo de conflictos, toma de decisiones colectivas, trabajo colaborativo en grupos, entre otros; además de fortalecer las habilidades comunicativas y argumentativas, afianzando la lengua materna, y en segunda instancia fortaleciendo un segundo idioma. Con estos elementos se elabora el producto final del ejercicio académico, con el cual se analiza la construcción de debates y/o 
consensos de criterios según la temática, debe señalarse que, el respeto por las ideas de los otros sujetos participantes, permiten la apropiación de los protocolos diplomáticos y de la investigación realizada por cada delegado representante en aspectos políticos, económicos, culturales, sociales, ecológicos y legales de cada nación.

Según (Gaete-Quezada, 2011:32) el juego de rol es una experiencia práctica con la cual el participante puede adquirir un aprendizaje significativo al momento de interpretar o representar acciones por medio de una simulación lúdica, para ello, se aplicó esta estrategia con el evento denominado: Simulación de la Asamblea de la Organización de las Naciones Unidas.

Por otra parte, la aplicación de diferentes estrategias por parte de los organizadores permite a los participantes, adquirir competencias y habilidades (García Ruíz, 2006:56), los cuales estimulan al delegado a interiorizar su papel dentro de la representación realizada.

En Bogotá, se ha practicado durante treinta años la estrategia pedagógica Simonu Bogotá, una propuesta que refiere a la simulación de las Naciones Unidas en diferentes escenarios académicos como colegios y universidades. El objetivo de esta estrategia es permitir el acercamiento entre los estudiantes y las instituciones públicas y privadas. Esta simulación gira en torno a la Carta de las Naciones Unidas, la Declaración Universal de los Derechos Humanos, el ABC de las Naciones Unidas y el ABC la enseñanza de los Derechos Humanos (SIMONU Bogotá, 2020).

Esta simulación académica, se ha replicado en varias Instituciones de Educación Superior en Colombia y a nivel internacional, de manera 
que se pueda proyectar el trabajo diplomático de las diferentes Naciones que se presentan para cada Objetivo de Desarrollo Sostenible.

\section{Marco teórico}

Los objetivos de desarrollo sostenible analizados en este apartado corresponden al ODS No. 13 Acción por el clima, seguido por los ODS No 1 Hambre cero y ODS 2. Fin de la pobreza:

\section{Acción por el clima. Objetivo No. 13}

El cambio climático es una preocupación que inquieta a los líderes globales, debido a la acción del ser humano sobre las dinámicas naturales presentadas en la última década, según el informe: Índices e indicadores de Desarrollo Humano del Programa de las Naciones Unidas para el Desarrollo - PNUD, (2018), el calentamiento global ha incrementado en un $1{ }^{\circ} \mathrm{C}$ por encima de los niveles preindustriales, este cambio repercute entre otros factores que afectan a la población, uno de ellos, es la salud de cada individuo; según las estadísticas presentadas en este informe, la esperanza de vida ha aumentado pero deja un sinsabor frente a la calidad de vida que van a tener las futuras generaciones.

La preocupación por el cambio climático, ha sido expresada por la sociedad civil, por varios gobernantes y por diferentes organismos internacionales como la las Naciones Unidas que desde los años 90 ha sido insistente en impulsar acciones para mitigar los cambios acelerados del clima global; sin embargo, los avances tecnológicos suscitados por las diversas etapas de la Revolución Industrial han coadyuvado al 
deterioro de los factores climatológicos, apoyados por el uso del carbón y los hidrocarburos como fuentes primarias generadoras de energía. (Rátiva Gaona, 2013:12).

El crecimiento económico experimentado durante el periodo de posguerra permitió que muchos individuos, agobiados por la desigualdad, superaran el umbral de la pobreza (Franco, 2013:45) y se insertaran en la dinámica competitiva (Ulloa, 2019:78). No obstante, las prácticas implementadas por el entorno político y empresarial distaban del equilibrio medioambiental y la explotación de los recursos naturales bajo condiciones idóneas, generando un modelo de alto impacto natural. Factores como la erosión costera, el derretimiento de los glaciares, el debilitamiento de los arrecifes de coral y el aumento del nivel del mar son problemas que preocupan a una sociedad comprometida con las generaciones venideras (Asociación Interamericana para la Defensa del Ambiente [AIDA], 2016:22).

Se estima que la proliferación de los gases de efecto invernadero y su vertimiento en la atmósfera acelerarán el deterioro de la capa de ozono, y es necesario plantear una acción conjunta de la comunidad internacional para mitigar tal preocupación (IBERDROLA, 2017:10).

Una de las actividades más cuestionadas la explotación de hidrocarburos bajo la técnica no convencional de fracturamiento hidráulico, conocida como fracking, caracterizada por la inyección masiva de agua y compuestos químicos para fragmentar, mediante un taladro, la roca madre que almacena el petróleo de esquisto, cuya profundidad oscila entre los 3000 y 6000 metros, distancia necesaria 
para coincidir con los acuíferos del subsuelo y liberar el gas metano a la superficie. Sin embargo, no hay consenso entre las comunidades respecto a la disposición final de los residuos procedentes de dicha actividad, es por esto, que se buscan mecanismos de participación ciudadana que propendan por generar una legislación acorde con los compromisos ambientales (Howarth, Ingraffea y Engelder, 2011:33).

Otra problemática, que afecta al objetivo No. 13 Acción por el clima, es la sobreexplotación de los bosques con miras a extender la frontera agrícola y ganadera, ante un crecimiento demográfico que ha sido acorde con la mejora en los indicadores de salud pública, la expectativa de vida al nacer e ingresos más altos para la clase media emergente. De acuerdo con los datos otorgados por la Organización de las Naciones Unidas para la Alimentación y la Agricultura - FAO, la perspectiva de crecimiento mundial para el 2050 será cerca del 30\% de la tasa actual, es decir, se proyecta una población cerca a los 10.000 millones, donde se debe trabajar en el acondicionamiento de los suelos y de los terrenos que son fértiles, se estima que, por cada ser humano se consume alrededor de 43,9 kilogramos por año, una cifra alarmante que impacta el equilibrio de los ecosistemas respecto a la desertificación del suelo, el uso intensivo de agua potable y los gases de efecto invernadero expulsados a la superficie (Organización de las Naciones Unidas para la Alimentación y la Agricultura [FAO], 2018:56).

Al mismo tiempo, la deforestación de grandes extensiones selváticas expone a la sociedad al calentamiento global, al limitar las posibilidades naturales de absorción del dióxido de carbono, el óxido de nitrógeno, 
metano, entre otros gases emisores de radiación que debilitan la capa de ozono (lbarra, 2012:43).

Por otra parte, la exposición directa a los rayos ultravioleta genera aumentos en la temperatura atmosférica y oceánica en los casquetes polares, el océano glacial ártico y antártico y sufran niveles de derretimiento superiores a la era preindustrial; cambios en los patrones de precipitación del agua, causando un aumento el nivel del mar e impacten de forma negativa la flora y fauna del área de influencia y sectores adyacentes (Comisión Económica para América Latina y el Caribe[CEPAL], 2018).

Gráfica 3 Efectos del cambio climático. Adaptado de (CEPAL, 2018)

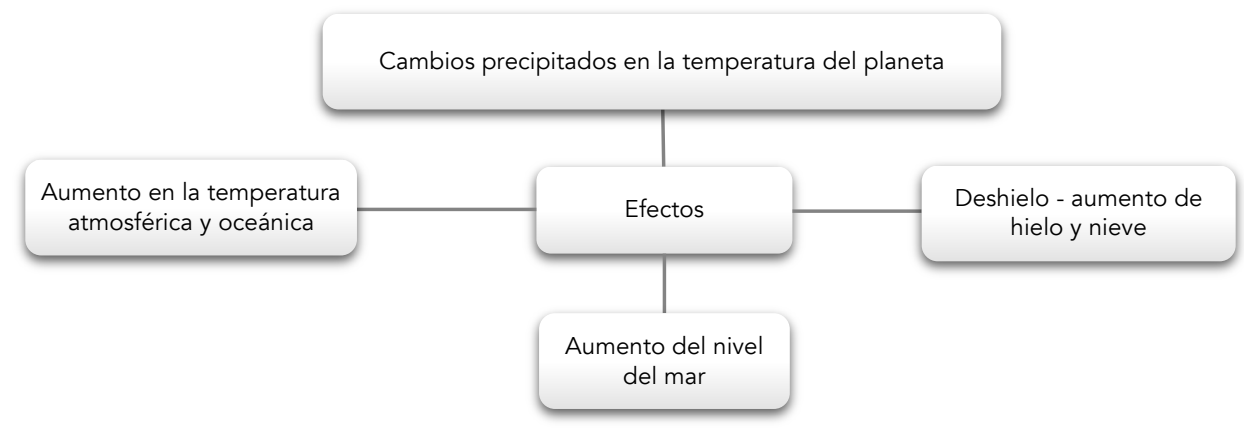

Asimismo, los Estados Insulares en desarrollo como Tuvalú, Kiribati, Nauru, Vanuatu y Las Maldivas, están perdiendo terreno debido a la ubicación que tienen con relación al nivel del mar, pese a que son territorios con niveles de contaminación bajos, sufren las consecuencias del crecimiento desbordado en otras latitudes, las consecuencias en corto plazo se prospectan frente a la desaparición 
física y jurídica como Estado por perder el atributo principal, su territorio, a causa de las inundaciones (Yasunaga-Kumano, 2016:72).

Frente a ello, la comunidad internacional se enfrenta a un nuevo capítulo concerniente a las migraciones, los refugiados climáticos, cuyos efectos se manifiestan en la pérdida de identidad cultural, la xenofobia, la carencia del libre desarrollo de la personalidad, la reducción de la tierra fértil para la agricultura y el acceso a fuentes de agua potable, entre muchas situaciones desconocidas hasta el momento (Agencia de las Naciones Unidas para los refugiados [ACNUR], 2018).

No obstante, los diferentes líderes mundiales han expresado su interés en mitigar los efectos causados durante la última década y analizan la implementación acciones de mejoramiento para mitigar el impacto que supone los cambios en los patrones de comportamiento ecosistémico. (Amnistía Internacional, 2018:36) Fruto de ello, la XXI Conferencia de las Naciones Unidas sobre el Cambio Climático, conocida como el COP 21 o Tratado de París, se convirtió en el compromiso más ambicioso de la historia moderna para frenar los embates de un sistema capitalista de consumo desbordado. Cada miembro constitutivo ha presentado un plan de acción enfocado en las emisiones de CO2, la deforestación, la explotación indiscriminada de los bancos de pesca, la radiación, entre un número indeterminado de problemáticas que afectan el entorno y la capacidad de adaptación de las comunidades más vulnerables.

El cumplimiento de la meta requiere que todos los gobiernos estén comprometidos, aunque existen limitantes como la no adhesión de un líder global en desarrollo industrial como los Estados Unidos de América al pacto celebrado en el año 2015; sin embargo, el avance en las 
negociaciones dependerá de la voluntad política de los representantes institucionales.

\section{Fin de la pobreza - ODS 1}

La pobreza extrema es la condición precaria en que se encuentra la sociedad al momento de no alcanzar a satisfacer las necesidades básicas de subsistencia como es el acceso al agua potable, a la educación de calidad, a las tecnologías de la información y la comunicación, a la vivienda digna y a la carencia de medios sanitarios básicos; problemática que han afectado a millones de personas durante lustros (Vargas Hidalgo y Martínez Austria, 2017-2018).

De acuerdo con los indicadores de (ACNUR, 2018:21), el umbral de pobreza está calculado en 1,90 dólares al día, es decir 57 dólares al mes y se calcula que existen 783 millones de personas en esta condición, una cifra preocupante para los representantes de gobierno de los países miembros de la ONU y que invita a reformular las políticas de estado, en pro de mitigar el impacto de las comunidades vulnerables, uno de los factores que más queja a esta población es que se encuentran expuestos a factores asociados como la proliferación de enfermedades, la desnutrición y el analfabetismo, entre otros.

Los mayores afectados por este flagelo son los niños y adolescentes quienes, en la mayoría de los casos, no reciben educación debido a que los padres destinan el dinero para otros gastos familiares; esta población es la más vulnerable y se estima que hay un $83 \%$ de la 
población infantil en 89 países en esta condición. Unicef en compañía del Grupo del Banco Mundial, han establecido comunicación con los diferentes gobiernos para evaluar los indicadores de pobreza infantil nacionales y fortalecer los sistemas de protección al menor garantizando los derechos fundamentales como es la educación y la salud para mejorar su calidad de vida (Fondo de las Naciones Unidas para la Infancia [UNICEF], 2019).

A su vez, los conflictos internos de cada país desembocan en guerras civiles, que influyen directamente en la manera de abordar la situación, debido al desplazamiento forzado y la migración de comunidades que impactan los niveles de población en las áreas urbanas. (Hobsbawm y Eguibar, 2007).

Manifestaciones populares como la "Primavera Árabe", acaecida a finales del año 2010 en países como Túnez, Libia, Egipto, Yemen y Siria, cuya finalidad se basaba en un cambio democrático pacífico no logró su cometido y desencadenó en una crisis migratoria sin precedentes hacia naciones de renta alta que se enfrentan a una problemática difícil de abordar. Estados europeos como Alemania han implementado sistemas flexibles para acoger los flujos de personas que huyen de su tierra natal, buscando mejores condiciones de vida para sus familias. Sin embargo, el proceso de adaptación ha sido impactado por la aversión al cambio, dado el caso que se enfrentan a la barrera cultural, mano de obra poco calificado y limitaciones idiomáticas, factores que coartan el acceso a puestos de trabajo intensivos en conocimiento (Peres Díaz, 2015:28).

Pese a lo anterior, una de cada 10 personas a nivel mundial vive en extrema pobreza, donde el $80 \%$ se localiza en el sur de Asia y África 
Subsahariana, regiones que gozan de niveles industriales bajos y cuya fuente de subsistencia se enfoca en la agricultura. Además, la irrupción democrática hace parte del diario vivir, como consecuencia de la incursión de grupos armados como Boko Haram y a la ausencia del estado central al momento de ejercer soberanía sobre las fronteras nacionales (PNUD, 2019).

Si bien las tendencias no son favorables al momento de interpretar la situación socioeconómica de la población afectada, es necesario tomar en cuenta que la técnica actual para la medición de la pobreza pone en escena la discusión de los indicadores, cuyo mecanismo divide los ingresos totales del hogar entre sus miembros. Frente a ello, el núcleo familiar con menores entre los 0 y 14 años va a ser propenso a un nivel de pobreza superior, debido al cálculo obtenido entre los generadores de ingresos y aquellos que no se encuentran en edad de trabajar. Por tal razón, la oferta adecuada de empleos de calidad coadyuva al mejoramiento de la calidad de vida de los ciudadanos, situación que debe ser afrontada mediante políticas públicas de largo plazo (Banco Mundial, 2016).

América Latina, es un territorio que no se escapa a las condiciones adversas de la pobreza, la privación de los niveles de bienestar sigue afectando a las generaciones venideras y los indicadores no han tenido incrementos significativos desde hace más de una década. Desde el año 2014, las condiciones precarias para sus habitantes se han visto impactadas al alza, después de un período marcada por los avances suscitados en la materia. Según estudios de la Comisión Económica para América Latina y el Caribe, organismo especializado de las 
Naciones Unidas para el desarrollo económico y social, 186 millones de personas $(30,7 \%)$ engrosaron las filas de pobreza, mientras que 61 millones de personas (10\%), cayeron en la pobreza extrema (Fundación BBVA Microfinanzas, 2018).

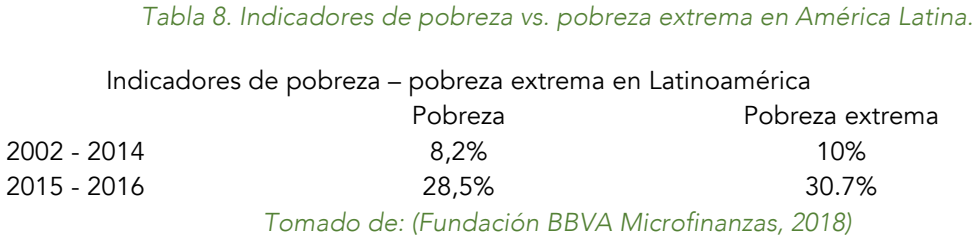

Los indicadores presentan la situación, frente a como mitigar la pobreza, para brindar soluciones duraderas a un problema que ha afectado a las sociedades modernas durante décadas, pero existen iniciativas que buscan brindar soluciones óptimas a través del acceso al microcrédito, el desarrollo de obras públicas, la educación financiera y la extensión agraria, conocida como la aplicación del conocimiento a las diversas actividades inherentes al agro. Al mismo tiempo, el crecimiento económico experimentando por los países de renta media ha permitido una masificación de los beneficios, pese a la brecha entre ricos y pobres que ha sido una constante durante décadas; países como China e India, quienes cuentan con el índice de población más alto del mundo, (Agencia Central de inteligencia $[\mathrm{CIA}], 2019)$ han visto prosperar sus economías impulsadas por el libre comercio, contribuyendo a la generación de riqueza nacional que ha sido invertida gracias a las políticas públicas que priorizan el gasto social (Acemoglu y Robinson, 2014:8).

Por último, las metas trazadas en el marco de los Objetivos de Desarrollo Sostenible, visión 2030, por los miembros de la Organización 
de las Naciones Unidas, buscan establecer mecanismos idóneos para abordar de una manera integral los desafíos de la pobreza extrema (FAO, 2018), además de factores asociados que impactan directamente los logros propuestos. Sin embargo, la ralentización económica que se ha vivido en los últimos años, fruto de las crisis financieras, ha generado que los avances sean lentos y los desafíos mayores, más aún, en aquellos países que no han logrado incursionar de manera efectiva en la corriente globalizadora (Naciones Unidas, 2017).

\section{Hambre cero. ODS No.2}

Erradicar el hambre en todas sus formas, afianzar la seguridad alimentaria y las condiciones de nutrición en el marco de la agricultura sostenible, son objetivos que se han trazado los gobiernos centrales para combatir el hambre extrema padecida por 795 millones de personas alrededor del mundo, cuya proyección de crecimiento se estima en 2000 millones para el año 2050, según datos de las Naciones Unidas (Naciones Unidas, 2019).

La problemática de la desnutrición presenta secuelas que causan la muerte de 7000 niños al día, debido a la escasez de alimentos; sin embargo, el contenido nutricional de las fuentes alimenticias puede carecer del componente adecuado para su desarrollo integral, poniendo de manifiesto la calidad de los productos consumidos. A su vez, las condiciones de salubridad y las enfermedades endémicas son factores asociados que aportan a la mortalidad en edad temprana. El Fondo de las Naciones Unidas para la Infancia UNICEF, estima que los 
niños que padecen de desnutrición aguda grave son 9 veces más propensos a morir que un niño en estado normal, debido al riesgo de enfermedades por las defensas débiles (UNICEF, 2019).

El embarazo es una etapa decisiva que puede limitar el desarrollo físico y cognitivo del embrión, bajo lo cual es necesario que las mujeres en gestación accedan a una alimentación adecuada para un buen desarrollo del feto, evitando secuelas como el nacimiento prematuro y el peso bajo. Según cifras del PNUD, 1 de cada 4 niños afronta problemas en la etapa de crecimiento, siendo el continente asiático el mayor contribuyente para impulsar las estadísticas al alza, con un $67 \%$ de los habitantes que enfrentan el hambre (Naciones Unidas para el Desarrollo, 2018).

Existen regiones que se han visto afectadas por el cambio climático lo ha acelerado el derretimiento de los glaciares y que provoca inundaciones y erosión costera que afecta el desarrollo de la agricultura. Más aún, la calidad del agua de irrigación y de consumo humano tiende a contaminarse por la salinización de las cuencas hidrográficas. Todos estos factores limitan las posibilidades de ser sostenibles en la política alimenticia, principio básico para el cumplimiento de los Objetivos de Desarrollo Sostenible visión 2030 (CEPAL, 2018).

Otro elemento que impacta de forma negativa las posibilidades de extender la frontera agrícola, con miras a satisfacer las necesidades alimenticias de una población creciente son los cambios de temperatura. En el caso de las olas de calor, la desertificación del suelo y la recuperación de los nutrientes se hace cada vez más notoria, mientras que las precipitaciones y las corrientes de frío modifican los procesos de adaptación de la flora y la fauna al nuevo paradigma natural (Comisión 
Europea, 2019), no obstante, pese a los desafíos climáticos que enfrenta la sociedad internacional, el foco central del problema no radica en la productividad sino en la dieta balanceada. No se trata de la cantidad de alimentos que son ingeridos diariamente, sino que se debe tomar en consideración la calidad de estos y su contenido nutricional, tanto las vitaminas y minerales, cuyos efectos van a modificar el porcentaje de valores diarios que requiere una persona para su normal funcionamiento, como es el caso de las mujeres embarazadas, cuya lactancia en condiciones normales beneficia el proceso de gestación (Rubio Vega, 2014:53).

Por otro lado, la inseguridad alimentaria centra su atención en la dificultad que presentan los individuos al momento de acceder a los bienes de consumo básico, donde los recursos económicos disponibles no son suficientes para adquirir los artículos de primera necesidad, y así desarrollar una dieta balanceada. Esto quiere decir que la ausencia de alimentos se hizo presente en algún momento del día o que ocurre de forma ocasional (FAO y otros, 2018).

\begin{tabular}{|c|c|c|c|}
\hline \multicolumn{4}{|c|}{ Inseguridad alimentaria } \\
\hline África & Asia & América Latina & América septentrional y Europa \\
\hline $22,3 \%$ & $7,3 \%$ & $7,6 \%$ & $1,5 \%$ \\
\hline $22,4 \%$ & $6,6 \%$ & $6,3 \%$ & $1,5 \%$ \\
\hline $25,4 \%$ & $6,5 \%$ & $7,6 \%$ & $1,2 \%$ \\
\hline $29,8 \%$ & $6,9 \%$ & $9,8 \%$ & $1,4 \%$ \\
\hline
\end{tabular}

La disposición de alimentos en la cantidad y la calidad requerida, sumado a las condiciones de salubridad adecuadas para la atención médica oportuna, son herramientas que permiten enfrentar un problema social que ha sido reducido en los últimos años, gracias a los 
avances tecnológicos y la cobertura mundial en salud en aquellas regiones que han desarrollado un proceso de crecimiento económico significativo en las últimas décadas, como resultado de la integración de mercados, pero que sigue afectando a un número significativo de personas que se encuentran bajo el umbral de pobreza (Brugué y otros, 2018).

\section{Metodología}

La Simulación de la Asamblea de la Organización de las Naciones Unidas, se realizó para un programa de Negocios Internacionales de una universidad privada, ubicada en Medellín, en el año 2018, la técnica didáctica aplicada para los dos eventos fue organizada por medio de un comité académico. Se utilizó una metodología cualitativa, descriptiva, con el fin de detallar la situación presentada en la representación y/o simulación de una asamblea, para ello se realizaron dos (2) eventos. 
Gráfica 1. Metodología de trabajo. Simulación de la Asamblea de Naciones Unidas. Elaboración propia. 2018

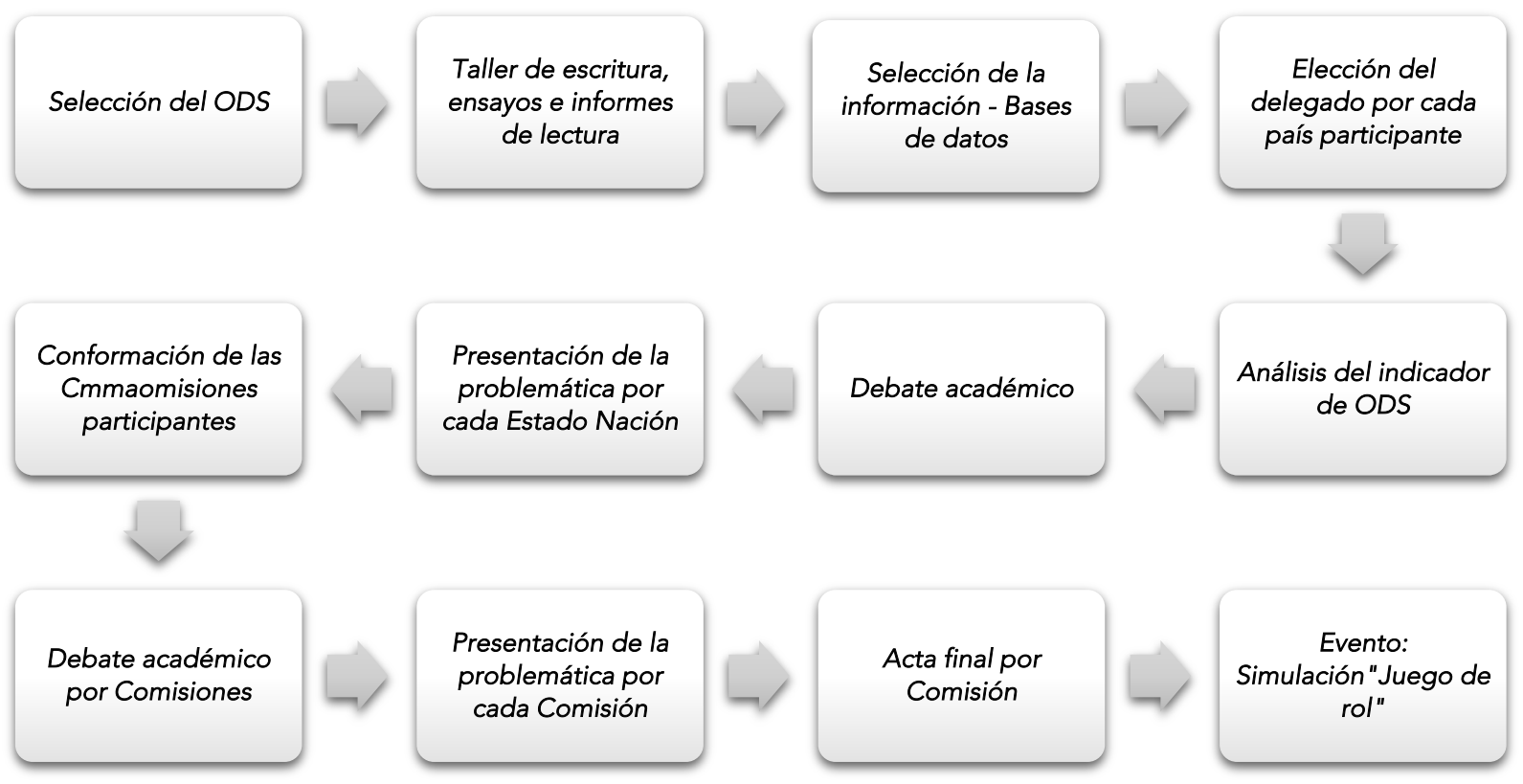

El Objetivo de Desarrollo Sostenible - ODS, analizado en el primer semestre académico de 2018, fue el No. 13. Acción por el Clima, con una participación de 35 delegados, de segundo y octavo semestre del programa de Negocios Internacionales, registrados en la asignatura de Diplomacia y Relaciones Internacionales, con el apoyo del programa de Permanencia académica de la misma Universidad, se brindó un taller sobre redacción, ensayos e informes de lectura, que fue la base para el inicio del proceso de formación, apoyados en los conceptos aplicados para el Modelo por Journoud y Landaluze, (2017).

La técnica aplicada fue la asignación de un país por cada delegado registrado, seguido por la investigación, análisis y construcción de su discurso frente a la situación que estaba presentando el país representado, posterior a esto se presentó un debate académico en el que se analizó la problemática de cada Nación. Una vez escuchados 
los argumentos de los delegados representantes, se conformaron las comisiones participantes para que entre los participantes se realizara la discusión académica frente a las dificultades encontradas, finalmente cada comisión presentó un producto final, relacionando las problemáticas y la resolución como postura académica.

En el segundo periodo del mismo año, se analizaron el objetivo ODS No. 1. Fin de la pobreza y el No. 2. Hambre cero con la participación de117 delegados de segundo y octavo semestre del programa de Negocios Internacionales, registrados en la asignatura de Diplomacia y Relaciones Internacionales. Estos delegados representaron 73 países. Se aplicó el mismo método de enseñanza para los dos eventos, la técnica aplicada en tres fases, y se presentaron las estrategias asociadas y la descripción relacionada a la evaluación del juego de rol:

Tabla 10. Fases de la Metodología aplicada. Elaboración propia. 2020

DESCRIPCION

\begin{tabular}{|c|c|c|}
\hline \multirow[t]{7}{*}{ Fase 1} & Lección magistral & Calidad de la exposición realizada por el docente/tutor \\
\hline & $\begin{array}{l}\text { Utilización de material de } \\
\text { apoyo }\end{array}$ & Utilización del material didáctico y de apoyo audiovisual \\
\hline & Análisis de documentos & Documentos y bases de datos oficiales \\
\hline & Trabajo independiente & Aprendizaje basado en el trabajo independiente \\
\hline & Trabajo en equipo & $\begin{array}{l}\text { Reuniones realizadas para debatir la información investigada y } \\
\text { trabajo colaborativo presentado. }\end{array}$ \\
\hline & Selección del ODS & Temática abordada de acuerdo a la realidad del país \\
\hline & Habilidad para la escritura & Escritura relacionada para la entrega del producto final \\
\hline \multirow[t]{4}{*}{ Fase 2} & Simulación & Representación de una situación real por medio de roles \\
\hline & Habilidades comunicativas & Uso adecuado del idioma y aplicación de un segundo idioma \\
\hline & Habilidad argumentativa & Producción de textos con características discursivas \\
\hline & Número de participantes & Número de participantes en el evento \\
\hline \multirow[t]{5}{*}{ Fase 3} & Evaluación del juego de rol & Análisis de la estrategia pedagógica aplicada \\
\hline & $\begin{array}{l}\text { Apropiación de los } \\
\text { conocimientos }\end{array}$ & $\begin{array}{l}\text { Aprendizaje de los conceptos diplomáticos y de los objetivos } \\
\text { de desarrollo sostenible }\end{array}$ \\
\hline & $\begin{array}{l}\text { Resolución y manejo de } \\
\text { conflictos }\end{array}$ & $\begin{array}{l}\text { Aprendizaje alcanzado frente a las actitudes tomadas en los } \\
\text { debates realizados }\end{array}$ \\
\hline & Toma de decisiones colectivas & Decisiones tomadas en los debates realizados \\
\hline & Producto final & Documento realizado para finalizar el juego de rol \\
\hline
\end{tabular}


Una vez realizadas las dos actividades académicas, se aplicó la rúbrica de evaluación para el juego de rol, por medio de entrevistas a los delegados participantes, teniendo en cuenta los criterios de valoración de las estrategias empleadas para la aprehensión del conocimiento de acuerdo con los parámetros establecidos en las directrices brindadas por el docente/tutor. Las preguntas tuvieron una valoración de escala de 1 a 5, siendo el primer la calificación más baja y el 5 como valoración superior $y$, en las preguntas finales, se plantearon peguntas abiertas para conocer las percepciones y opiniones frente al desarrollo de las actividades.

La búsqueda de información secundaria, se realizó por medio de las bases de datos oficiales de las Naciones Unidas como Sistema de información bibliográfica de la ONU (UNBisnet), documentos de las Naciones Unidas SAD, base de datos de la Comisión Económica y Regional para América Latina y el Caribe (CEPAL), Colección de tratados de las Naciones Unidas (UN Treaty), Banco Mundial, Organización de las Naciones Unidas (ONU), Google Académico, Ebsco, UN Data y páginas oficiales de las delegaciones de cada Estado Miembro ante la ONU.

\section{Resultados}

En el primer evento de la Simulación de la Asamblea, se desarrolló en el primer semestre académico del 2018, para este evento participaron 35 delegados, una vez se terminó de aplicar la técnica del juego de simulación, se recopilaron los siguientes datos: 
En la fase 1 del primer evento, se tuvieron en cuenta las habilidades y conocimientos adquiridos para trabajar la simulación. El objetivo de desarrollo sostenible elegido correspondió al ODS No. 13. Acción por el clima, valorado con la calificación de (5) con un 82,9\% de aprobación, seguido de las orientaciones brindadas por el docente/tutor alcanzando un $60 \%$ en la escala (4), esta persona se apoyó en el uso de material audiovisual, consiguiendo una aprobación del 48,6\%, en la escala (5).

Una vez realizado el análisis de documentos de la primera parte arroja una calificación de desaprobación, alcanzando el mayor porcentaje en la valoración (3) con un 71,4\%, seguido de la valoración (4) en 22,9\%; mientras que el trabajo independiente, presenta en la valoración (2) un $40 \%$ de desaprobación y en el trabajo en equipo se alcanza en la valoración (4) un 74,3\%. Las habilidades escriturales fueron valoradas en la escala (3) con un $40 \%$.

Gráfica 4. Fase 1 - Preparación juego de rol.

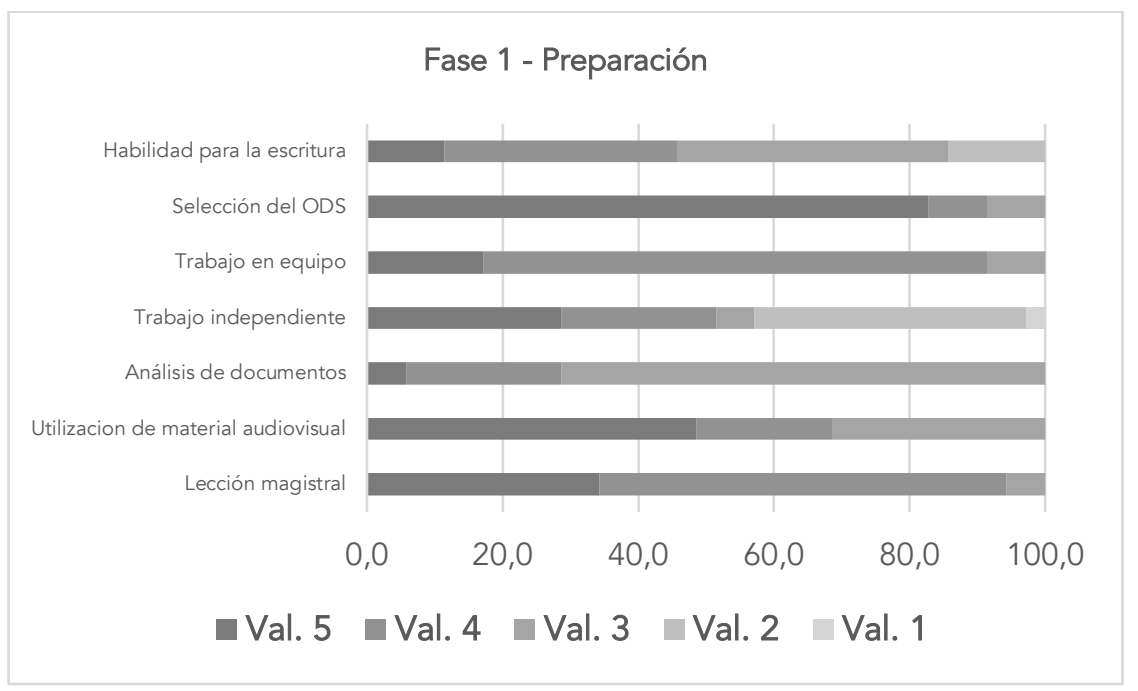


En la segunda fase, se evaluó el juego de rol aplicado en la simulación realizada, alcanzado una valoración en la escala (4) con un $54,29 \%$, el mejoramiento de las habilidades comunicativas se evalúa en la escala (3) con un $\mathbf{7 7 . 1 4 \% ~ y ~ l a s ~ h a b i l i d a d e s ~ a r g u m e n t a t i v a s ~ c o n ~ u n ~}$ $45.71 \%$, y el $37,14 \%$ valoró en la escala (5) el número de participantes en el evento.

Gráfica 5. Fase 2 - Simulación juego de rol.

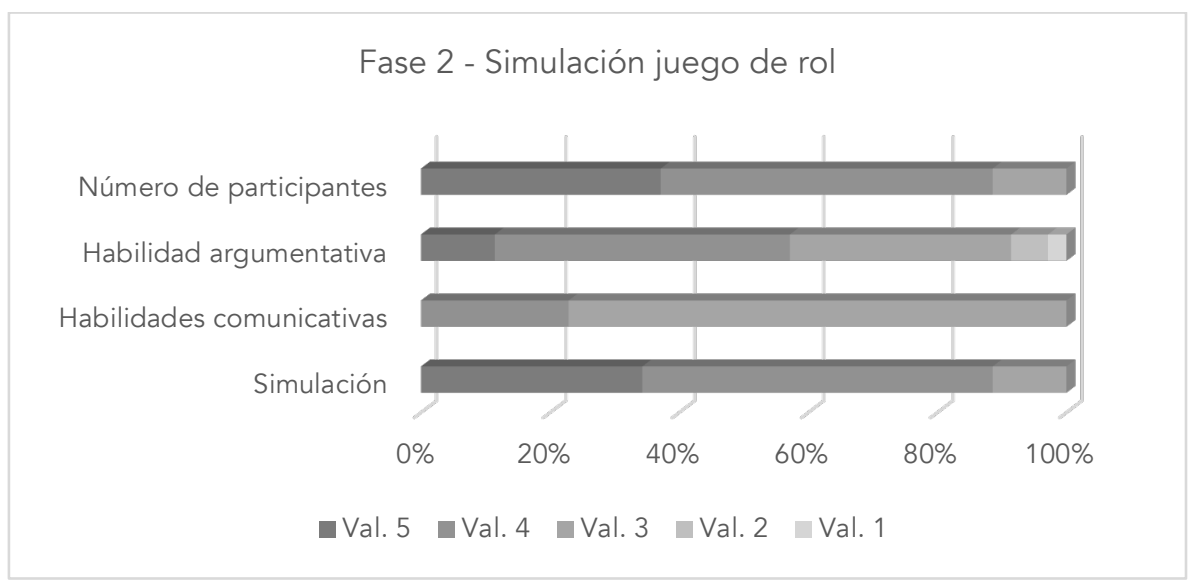

La tercera fase, se realiza el cierre del evento y de la aplicación del juego de simulación y se analiza la evaluación del evento con una valoración en (3) de 45,7\% seguido de la (4) con un 42,9\%., frente a la apropiación de los conocimientos referente a los ODS, se encuentra una calificación (5) con un $40 \%$ y en el aprendizaje y mejoramiento de las habilidades frente a la resolución y manejo de conflictos, es la escala (4) con un $65,7 \%$, toma de decisiones (3) con un $42,9 \%$ y frente al trabajo entregable final, se evalúa en la escala (2) con un $57,1 \%$. 
Gráfica 6. Fase 3. Evaluación

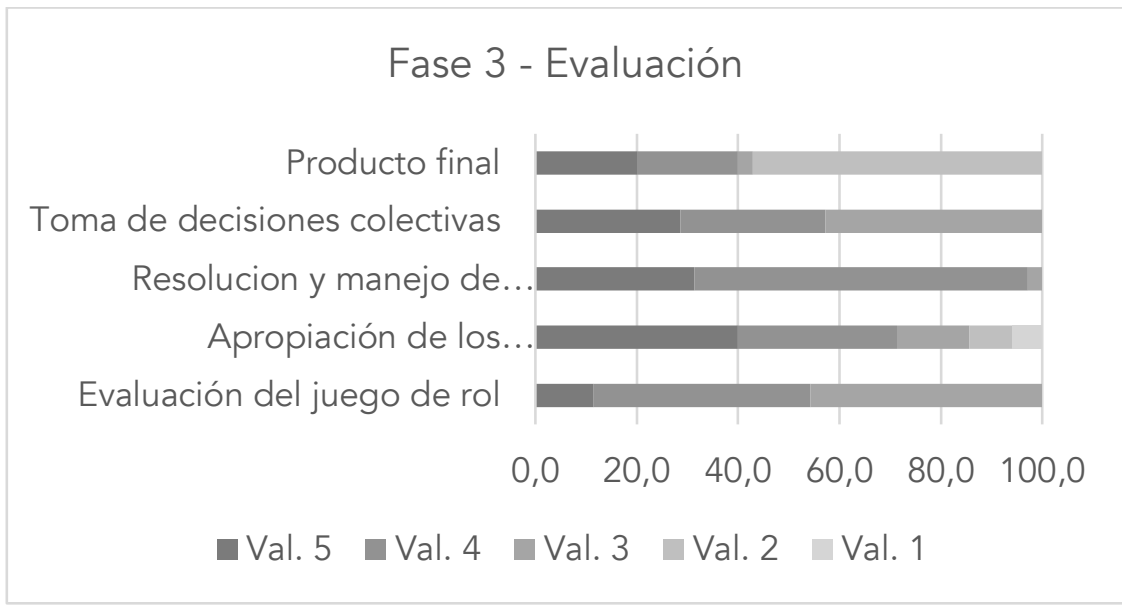

El revisar los resultados del primer evento, se discuten con los participantes los resultados, a través de una rúbrica de evaluación para determinar los aspectos positivos y los aspectos por mejorar. Se encuentra que el programa académico, no había realizado en ocasiones anteriores juegos de simulación aplicados y con público presente, entonces la aprensión de los conocimientos y de la metodología al ser diferentes, fueron tomadas por los participantes como un reto difícil de afrontar.

Esto se demuestra con el trabajo independiente realizado, el cual presenta un porcentaje alto (40\%) de rechazo, escala (2), gracias a que se debía realizan investigación de información de cada país, en fuentes oficiales y apropiarse de dichos conocimientos y a su vez, realizar un trabajo escrito de manera grupal donde se debían evaluar las competencias escriturales. En la segunda fase, los participantes se 
encuentran ante un reto de la aplicación de las habilidades comunicativas. Inicialmente se propuso trabajar en español y en lenguas extranjeras, se introdujo el inglés, francés y portugués, la participación fue baja, escala (3) con un 77.14\%, las apreciaciones estuvieron orientadas al nerviosismo y pánico escénico que produce hablar ante un público, incrementando el nivel de desconfianza para expresarse en otro idioma; en la etapa final, los participantes informan que al solicitar el trabajo elaborado por el grupo y entregado de manera escrita, con indicaciones expresas de citación según las normas APA y con información real de la situación de cada país, hizo que la presión aumentara, debido a que el tiempo de elaboración y de consulta fue desarrollado en 4 semanas.

La apropiación del conocimiento respecto al abordaje en la aprehensión de la información relacionada con el ODS fue valorada con un $40 \%$.

La segunda Simulación de la Asamblea General de la Organización de las Naciones Unidas, se realizó en el mismo año (2018) en el segundo semestre académico, con el triple de participantes (117) y se trabajaron dos temáticas por el número de delegados participantes. (ODS 1 y 2), para ello, se analizaron los resultados del primer evento y se realizaron ajustes metodológicos.

De las siete variables analizadas, seis alcanzaron la valoración más alta (5): la selección de dos ODS, con un 95,7\% de aceptación, la lección magistral realizada por el docente/tutor 48,7\%, asimismo la utilización del material audiovisual con un 83.8\%, el análisis de documentos con un $31.6 \%$, trabajo en equipo con un $85,5 \%$ y 
habilidades para la escritura con un 40,2\%. El trabajo independiente se valoró en la escala (4) con un 58.1\%.

Gráfica 7. Fase 1 - Preparación juego de rol.

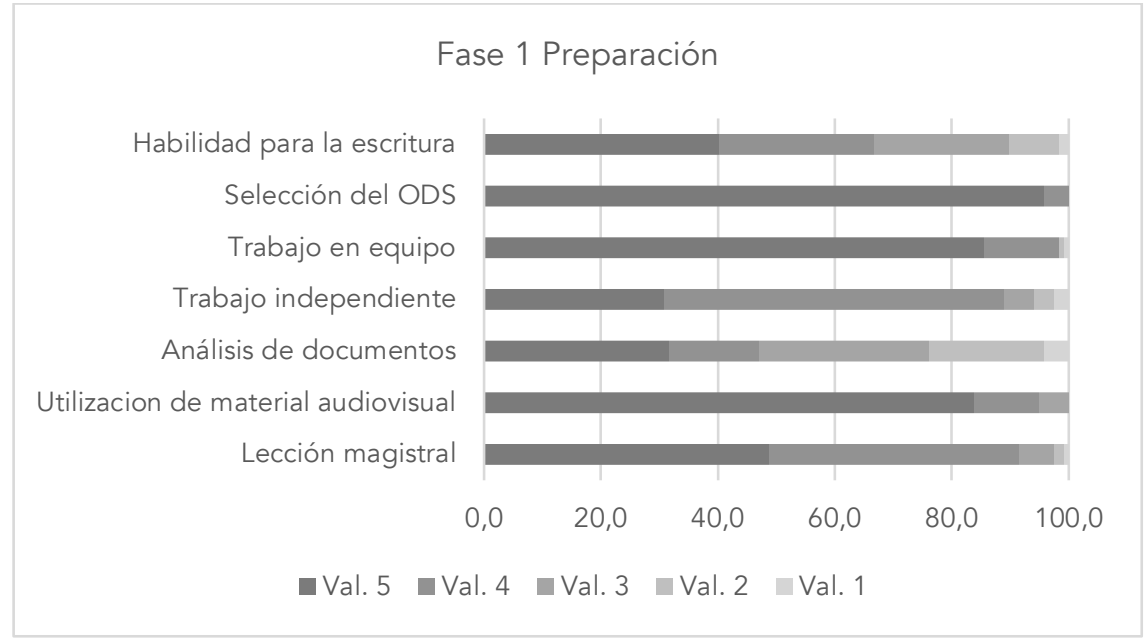

La segunda fase, fue evaluada con la escala (5), la simulación del evento con un $86.3 \%$, el número de participantes con un $86.3 \%$ y el mejoramiento de las habilidades comunicativas 94\% y habilidades argumentativas con un $61,5 \%$.

Gráfica 8. Fase 2 - Simulación juego de rol.

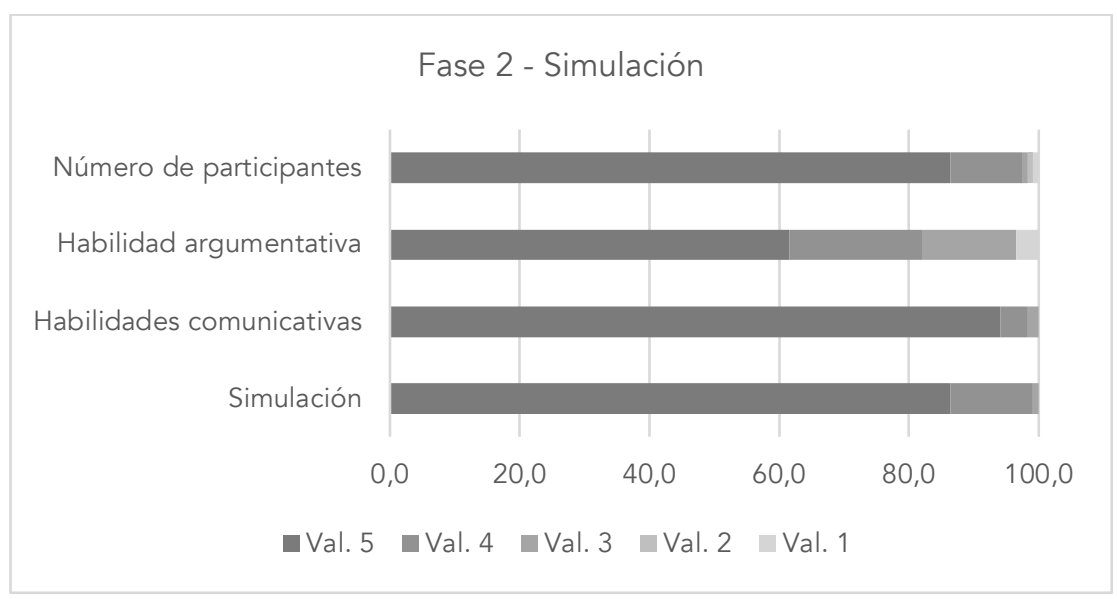


La última fase, relacionada con la evaluación del juego de simulación, se valoró en la escala (5) con un $88.9 \%$, la apropiación de los conocimientos con un $74.4 \%$, la resolución y manejo de conflictos con un $56.4 \%$ y la toma de decisiones colectivas valorada en un $60,7 \%$. El producto final para entregar como resultado de la estrategia se valoró en $74.4 \%$.

Gráfica 9. Fase 3. Evaluación

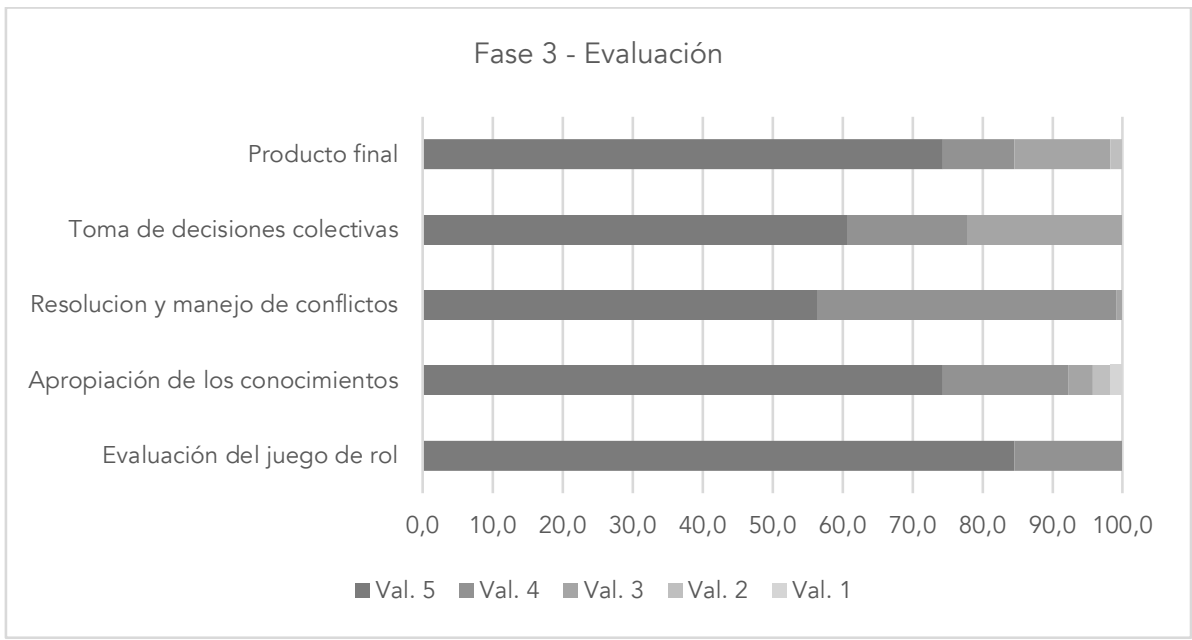

Con los resultados del primer evento, se aplicaron acciones de mejoramiento, que permitieron comprender la experiencia del juego de simulación por parte de los participantes. Los aspectos

Trabajo independiente pasó de la escala de valoración (2 - 40\%) a la (4-68\%), gracias a la planificación de las actividades a entregar con un incremento del tiempo para dedicar a las actividades a desarrollar y del acompañamiento por parte del docente/tutor; otro de los aspectos que mejoró en los resultados obtenidos, correspondió al análisis de documentos (3) con 71,4\% pasando a la escala (5) con un $37 \%$ y la habilidad para la escritura (3) $40 \%$, pasó a la escala (5) con un $47 \%$, las acciones de mejoramiento estuvieron relacionadas con la 
inclusión de bienestar institucional con capacitaciones presenciales y virtuales para el desarrollo de dichas habilidades.

La exposición realizada por el docente/tutor, orienta al participante, en el objetivo que se quiere lograr con la investigación por país, teniendo en cuenta las orientaciones que la Organización de las Naciones Unidas tiene contempladas tales como la participación activa con voz y voto de las 193 Estados Miembros, y siguiendo la normativa contemplada en el Manual para las Delegaciones (ONU, 2018). En el segundo evento, se implementó la intervención activa de los participantes, que además de mejorar las habilidades comunicativas, pudo evidenciar la habilidad argumentativa puesto que por medio de un documento (tipo acta), los participantes expusieron las problemáticas presentadas por cada Estado Nación y la postura de los delegados ante dicha situación.

Este apoyo institucional, también aportó al mejoramiento de la actividad en la segunda fase, el fortalecimiento de las habilidades comunicativas y las habilidades argumentativas, estuvieron en la escala 3 con un $77 \%$ y $44 \%$ respectivamente, pasando a la escala (5) con un $61 \%$ y $86,3 \%$.

Frente a las habilidades comunicativas y argumentativas, se implementaron ensayos con micrófonos y de manejo del escenario para disminuir el pánico escénico, se estimularon a los demás participantes para que adquirieran conocimientos en otros idiomas, incluso en un nivel básico, para que realizaran en la ponencia una participación corta. Este encuentro se desarrolló en (9) idiomas: alemán, catalán, coreano, francés, inglés, hebreo, portugués, italiano, mandarín, y se realizó una interpretación en lengua de señas colombianas. La valoración se 
incrementó de 77,14\% en la escala 3, a la escala 5 con un 94\% de aceptación. La apropiación de los conocimientos fue valorada con un $74.4 \%$, gracias a las estrategias antes mencionadas, que permitieron que los participantes pudieran estar más involucrados y a su vez interiorizar cada objetivo desarrollado, frente al porcentaje obtenido del primer evento, se incrementó en 34 puntos, esto permite que los eventos, junto con las estrategias planteadas, cuenten con un mejoramiento continuo para alcanzar niveles más altos.

\section{Conclusiones}

Los Objetivos de Desarrollo Sostenible ODS, tienen una visión holística de las problemáticas mundiales, tales como el cambio climático y la desigualdad económica, entre otros; el éxito del cumplimiento mundial es la vinculación de cada gobierno, empresa privada y pública y de cada uno de los seres humanos que interiorice su accionar como réplica de las dificultades que presenta el planeta y así mejorar de manera sistemática la permanencia de los hombres en generaciones venideras. El crecimiento económico de cada Nación está en pro de reducir los índices de pobreza y mejorar o alcanzar los niveles de igualdad en las personas

La aplicación de la estrategia metodológica, con la técnica de juego de simulación permite que los participantes mejoren sus habilidades y competencias en la aprehensión de las ideas, conceptos relacionados y aprehensión de los ODS, para este caso, en las negociaciones 
internacionales, los aspectos diplomáticos y los objetivos de desarrollo sostenible, que orientan a cada una de las naciones elegidas.

La oportunidad de realizar estos eventos permite que se puedan hacer ajustes con el fin de mejorar los resultados e incrementar los elementos pedagógicos y metodológicos para hacer una experiencia de conocimiento para el programa.

Los resultados de la estrategia pedagógica materializada a través del juego roles demuestran que los participantes logran comprender la realidad que aqueja a la sociedad internacional, analizar las problemáticas presentadas en cada Estado Nación, comprender y sistematizar la información que se presenta y está a la vanguardia, entender la función diplomática que se desarrolla a través de las relaciones internacionales y cómo esta agenda es manejada por los organismos mundiales; así mismo, analizar la realidad de las naciones frente a las problemáticas globales representadas a través de los ODS.

\section{Referencias}

Acemoglu, D., y Robinson, J. A. (2014). Por qué fracasan los países. Los orígenes del poder, la prosperidad y la pobreza. Revista Austral de Ciencias Sociales, 26, 139-146. Disponible en http://mingaonline.uach.cl/pdf/racs/n26/art08.pdf

ACNUR. (2018). Agencia de las Naciones Unidas para los refugiados. Nueva York: Naciones Unidas.

Disponible en https://www.acnur.org/noticias/press/2018/12/5c17d5534/estados-alcanzan-unhistorico-acuerdo-para-los-refugiados-y-se-comprometen.html

ACNUR. (10 de 2018). Pobreza extrema, ¿qué podemos hacer para acabar con ella? Disponible en https://eacnur.org/es/actualidad/noticias/eventos/pobreza-extremaque-podemos-hacer-para-acabar-con-ella

AIDA. (01 de 2016). Asociación Interamericana para la Defensa del Ambiente. Disponible en https://aida-americas.org/es/blog/los-arrecifes-de-coral-y-los-impactos-imprevistosdel-turismo 
Aizen, M. (2017). El portazo de Estados Unidos al Acuerdo de París: un ruido que no se escuchó. Nueva Sociedad, 271, 45-54.

Amnistía Internacional. (02 de 2018). Soluciones a la crisis mundial de refugiados. Disponible en https://www.amnesty.org/es/latest/campaigns/2015/10/eight-solutions-worldrefugee-crisis/

Banco Mundial. (06 de 2016). Banco Mundial. Disponible en http://www.bancomundial.org/es/news/feature/2016/06/29/ninos-america-latina-sonmas-afectados-por-pobreza

Brugué, Q., Gomá, R., y Subirats, J. (2018). De la pobreza a la exclusión social. Nuevos retos para las políticas públicas. Revista Internacional de Sociología, 60(33), 7-45.

CEPAL. (2018). Guía Metodológica. Planificación para la implementación de la Agenda 2030 en América Latina y el Caribe. Santiago: Naciones Unidas.

). (2018). La economía del cambio climático en América Latina y el Caribe. Santiago: Naciones Unidas. Disponible en https://repositorio.cepal.org/bitstream/handle/11362/42228/4/S1701215A_es.pdf

CIA. (28 de 02 de 2019). Agencia Central de Inteligencia. Disponible en https://www.cia.gov/library/publications/the-world-factbook/

Comisión Europea. (20 de 02 de 2019). Consecuencias del cambio climático. Disponible en https://ec.europa.eu/clima/change/consequences_es

Documento CONPES. (2018). Documento CONPES Objetivos de Desarrollo Sostenible. Bogotá: Gobierno de Colombia.

Fajardo del Castillo, T. (2018). El Acuerdo de París sobre el cambio climático: sus aportaciones al desarrollo progresivo del derecho internacional y las consecuencias de la retirada de los Estados Unidos. Revista Española de Derecho Internacional, 23-52.

FAO. (2018). El estado de los bosques del mundo. Las vías forestales hacia el desarrollo sostenible. Licencia: CC BY-NC-SA 3.0 IGO.

(_ (2018). Perspectivas alimentarias. Resúmenes de mercado. Organización de las Naciones Unidas para la alimentación y la agricultura. doi:ISSN 1564-2801

FAO, FIDA, UNICEF, PMA y OMS. (2018). El estado de la seguridad alimentaria y la nutrición en el mundo. Fomentando la resiliencia climática en aras de la seguridad alimentaria y la nutrición. Roma: FAO.

Franco, R. H. (2013). Las clases medias en América Latina. Mexico D.F.: Siglo XXI. Disponible en https://repositorio.cepal.org/bitstream/handle/11362/2023/S3014C6142010_es.pdf?s equence=1\&isAllowed=y

Fundación BBVA Microfinanzas. (01 de 2018). Evolución de la pobreza en América Latina. Disponible en http://www.fundacionmicrofinanzasbbva.org/evolucion-la-pobrezaamerica-latina/

Gaete-Quezada, R. (2011). El juego de roles como estretegia de evaluación de aprendizajes universitarios. Educación y educadores, 14, No. 2, 289-307. doi:ISSN 0123-1294

García Ruiz, M. (2006). Las competencias de los alumos universitarios. Revista Interuniversitaria de formación del profesorado. doi:ISSN 0213-8646

Hobsbawm, E. J., y Eguibar, B. (2007). Guerra y paz en el siglo XXI. Barcelona: Crítica. 
Howarth, R. W., Ingraffea, A., y Engelder, T. (2011). Natural gas: Should fracking stop? Nature, 477, $271 . \quad$ Disponible en http://www.chriscunnings.com/uploads/2/0/7/7/20773630/fracking_yes_or_no.pdf

Ibarra, A. (2012). El problema. En Cambio climático y justicia ambiental. Bogotá: ILSA, Instituto para una sociedad y un derecho alternativo. Disponible en "http://biblioteca.clacso.edu.ar/Colombia/ilsa/20170809040228/pdf_996.pdf"

IBERDROLA. (2017). Informe de gases de efecto invernadero 2017. Madrid: IBERDROLA. Disponible https://www.iberdrola.com/wcorp/gc/prod/es_ES/sostenibilidad/docs/Informe_GEl.p df

Journoud, A., y Landaluze, I. (2017). El modelo de Simulación de Naciones Unidas como método de aprendizaje y evaluacuón de Derecho Internacional Público. Nueva York: ONU.

Mendoza, I. X. (2009). Actividades extracurriculares: un camino eficaz para fortalecer el conocimiento en la práctica universitaria. Laberinto, 9, 7-9.

Naciones Unidas. (10 de 2017). 30 años de lucha contra la pobreza (con resultados). Disponible en https://www.un.org/development/desa/es/news/social/internationalday-address-poverty.html

). (03 de 2019). Alimentación. Disponible en https://www.un.org/es/sections/issuesdepth/food/index.html

Naciones Unidas para el Desarrollo. (2018). Indices e indicadores de desarrollo humano: actualización estadística 2018. Nueva York: Naciones Unidas. Disponible en http://hdr.undp.org/sites/default/files/2018_summary_human_development_statistical _update_sp.pdf

ONU. (2018). Manual para las delegaciones. Septuagésimo tercer periodo de sesiones de la Asamblea General de las Naciones Unidas. Nueva York: ONU.

Organización de las Naciones Unidas para la Alimentación y la agricultura. (26 de 04 de 2018). Disponible en www.anpad.or.br/o

Perales, J. A. (2017). ¿Más y mejor ayuda?: la Declaración de París y las tendencias en la cooperación al desarrollo. Anuario Ceipaz, 71-102.

Peres Díaz, M. (2015). La "Primavera Árabe" y las nuevas tecnologías: consideraciones preliminares para la consolidación democrática. Entelequia(18). Disponible en https://revistaentelequia.wordpress.com/2015/04/13/la-primavera-arabe-y-las-nuevastecnologias-consideraciones-preliminares-para-la-consolidacion-democratica/

PNUD. (10 de 10 de 2017). Programa de las Naciones Unidas para el desarrollo. ) (2018). Indices e indicadores de Desarrollo Humano. Actualización estadística. Nueva York: Programa de las Naciones Unidas para el Desarrollo. Disponible en http://hdr.undp.org/sites/default/files/2018_human_development_statistical_update_ es.pdf

). (22 de 03 de 2019). Fin de la pobreza. Disponible en https://www.undp.org/content/undp/es/home/sustainable-development-goals/goal1-no-poverty.html

Rátiva Gaona, S. (2013). Pobreza, ambiente y cambio climático. Buenos Aires: CLACSO. 
doi:ISBN 978-987-1891-40-5

Revista para el desarrollo social. (02 de 20 de 2019). PNUD. Disponible en http://www.revistaparaeldesarrollosocial.org/temas/personas/hambre-cero/

Rubio Vega, B. A. (2014). El dominio del hambre. Crisis de hegemonía y alimentos. Mexico: Juan Pablos Editor.

SIMONU Bogotá. (04 de 02 de 2020). SIMONU Bogotá. Disponible en https://nacionesunidas.org.co/simonu/que-es/

Ulloa, F. (2019). Colombia: el tortuoso calvario de la transicion política hacia la paz. . El momento político de América Latina, 207.

UNICEF. (02 de 03 de 2019). Qué es la desnutrición. Disponible en https://www.unicef.es/noticia/que-es-la-desnutricion

). (27 de 03 de 2019). UNICEF. Disponible en https://www.unicef.es/prensa/casi-385millones-de-ninos-viven-en-situacion-de-pobreza-extrema-segun-un-estudio-conjunto

United Nation Climate Change . (11 de 2016). United Nation Climate Change . Disponible en https://www.ilo.org/aids/Publications/WCMS_193947/lang--es/index.htm

Vargas Hidalgo, A., y Martínez Austria, P. (2017-2018). El agua, el ingreso y la pobreza. AquaLAC, 10, $97 . \quad$ Disponible en http://www.unesco.org/new/fileadmin/MULTIMEDIA/FIELD/Montevideo/pdf/09Varga s.pdf

Yasunaga-Kumano, M. (2016). Los pequeños Estados Insulares en Desarrollo y los desplazados climáticos. Instituto Español de Estudios Estratégicos. Boletin ieee., 497511. Disponible en https://dialnet.unirioja.es/servlet/articulo?codigo=6029281 